\title{
Acceleration and deceleration of convoy electrons in grazing-ion-surface collisions
}

\author{
M. S. Gravielle and J. E. Miraglia \\ Instituto de Astronomía y Física del Espacio, Casilla de Correo 67, Sucursal 28, 1428, Buenos Aires, Argentina \\ and Departamento de Física, Universidad Nacional de Buenos Aires, Buenos Aires, Argentina
}

(Received 4 December 2002; published 15 April 2003)

\begin{abstract}
Convoy-electron emission produced by grazing-ion-surface scattering is studied in the framework of the distorted-wave theory. We develop a model, here named field distorted-wave (FDW) approximation, to describe the effect of the surface interaction on the electronic transition. In the model, the action of the surface field on the ejected electron is seen as an additional momentum transfer that depends on the projectile position. We apply the FDW approximation to analyze electron distributions for $100 \mathrm{keV}$ protons impinging on $\mathrm{LiF}(100)$ and $\mathrm{Al}(111)$ surfaces, which are insulator and metal materials, respectively. In the case of metals, the dynamic screening of the projectile is included in the Jost function corresponding to the final state. As experimentally observed, energy spectra of forward-ejected electrons display a prominent structure associated with the convoy-electron emission. We find that the maximum of the convoy-electron distribution is decelerated for LiF and accelerated for $\mathrm{Al}$, with respect to its position in ion-atom collisions, in quantitative agreement with the experimental data.
\end{abstract}

DOI: 10.1103/PhysRevA.67.042901

PACS number(s): 34.50.Dy, 34.50.Bw

\section{INTRODUCTION}

Electron emission produced during the grazing scattering of fast ions from solid surfaces has been the subject of intense research in the past years $[1-13]$. Due to the long interaction time of the projectile with the surface involved in such collisions, this is a powerful tool to study surface features. In particular, angular and energy spectra of emitted electrons provide relevant information about the atomic and electronic structure of the topmost layer of the solid.

One of the most interesting regions of electron observation angles is around the direction of specular reflection of the projectile. In this angular region, the energy distribution of the ejected electrons displays a prominent structure, whose shape and position differ markedly from that observed in ion-atom collisions. In this case, the experimental spectra show a cusp-shaped peak at electron velocities $\vec{k}_{f}$ close to the projectile velocity $\vec{v}$ [14], which is usually named capture to the continuum or convoy-electron peak (CEP). The formation of this peak is associated with electrons that recede from the target in close spacial correlation with the projectile, strongly interacting with its Coulomb potential. Then, the position and shape of the CEP are governed by the final Coulomb projectile-electron interaction $[15,16]$.

For glancing-angle ion-surface scattering, the CEP appears appreciably broadened and shifted with respect to its position in ion-atom collisions. Both features are a direct consequence of the presence of an effective surface interaction $[3,11]$. The sign of the energy displacement of the CEP depends on the electronic structure of the solid. For metals and semiconductors the peak is shifted to higher velocities $k_{f}>v$ [1-6], signifying an acceleration of the convoy electrons caused by repulsion of the induced surface potential. For insulator surfaces, instead, the dynamic response of the surfaces is expected to be weak, but the target ionization produced along the ion path originates a track potential which affects the emitted electrons. The convoy electrons are decelerated by the track potential, and the CEP has its maximum at lower velocities $k_{f}<v[7,17]$.

The aim of this work is to describe the energy distribution of convoy electrons originated by fast grazing-ion-surface collisions. For glancing angles and high impact energies, the emission of convoy electrons is primarily due to direct ionization of the surface atoms, while the contribution of the valence band (free-electron gas) is negligible around the CEP, even for metal surfaces $[11,13]$. In a previous article [18] we have studied the inner-shell emission from metal surfaces by employing a semiclassical formalism, in which the multiple collisions of the incident ion with the surface atoms are treated as single encounters with outermost atoms along the projectile path. In the model, the emission probability per unit path is expressed in terms of atomic probabilities, depending on the modulus and orientation of the impact parameter. In Ref. [18] the atomic ionization probability was evaluated with the continuum-distorted-waveeikonal-initial-state (CDW-EIS) approximation, without including the induced surface potential. Since this last interaction plays an important role in the emission of convoy electrons, our present goal is to develope a distorted-wave theory that describes the atomic ionization process in presence of a time-dependent external potential, as that originated by the surface. Such a model will allow us to give an account of the effect of the surface interaction on the electronic transition in collisions involving solid surfaces.

The work is organized as follows. In Sec. II A, we derive a distorted-wave formalism to deal with the atomic ionization process in presence of a time-dependent external field. In Sec. II B, the proposed model is applied to describe the electron emission in collisions with surfaces. Energy spectra of emitted electrons at the specular reflection direction are shown and discussed in Secs. III A and III B, for insulator and metal surfaces, respectively. In both cases, the energy shifts of the CEP are compared with recent experimental data. Section V contains our conclusions. Atomic units are used unless otherwise stated. 


\section{THEORETICAL MODEL}

\section{A. Atomic ionization in the presence of an external potential}

We consider a projectile $P$, with charge $Z_{P}$, impinging with velocity $\vec{v}$ on a target atom composed by a nucleus $T$ of charge $Z_{T}$ and an active electron $e$. As a consequence of the collision, the electron $e$ initially bounded to the target is ionized, and the process occurs in the presence of a timedependent potential $V_{0}(t)$. The frame of reference is fixed to the nucleus target, and since the target recoil is small, neglecting it does not affect the electron emission spectrum. We indicate with $\vec{r}_{T}\left(\vec{r}_{P}\right)$ the position vector of the electron $e$ with respect to $T(P)$, and with $\vec{R}_{T}$ the position vector of $P$ (with respect to $T$ ).

Employing the straight-line version of the impact parameter approximation, the projectile position reads

$$
\vec{R}_{T}(t)=\vec{\rho}+\vec{v} t
$$

with $\vec{\rho}$ being the impact parameter and $\vec{\rho} \cdot \vec{v}=0$. The electronic time-dependent Schrödinger equation to be solved is

$$
\left(H_{e l}-i \frac{\partial}{\partial t}\right) \Psi_{j}^{ \pm}\left(\vec{r}_{T}, t\right)=0, \quad j=i, f,
$$

where $\Psi_{j}^{ \pm}\left(\vec{r}_{T}, t\right)(j=i, f)$ are the initial and final electronic wave functions, and $H_{e l}$ is the electronic Hamiltonian

$$
H_{e l}=K_{e l}+V_{T}+V_{P}+V_{0}(t)
$$

with $K_{e l}=-1 / 2 \nabla_{\vec{r}_{T}}^{2}$ being the electron kinetic-energy operator and $V_{T}=-Z_{T} / r_{T}\left(V_{P}=-Z_{P} / r_{P}\right)$ being the $T-e(P-e)$ Coulomb interaction. When the colliding particles are far away from each other, the initial $\phi_{i}^{+}$and final $\phi_{f}^{-}$collision states satisfy

$$
\left(H_{j}-i \frac{\partial}{\partial t}\right) \phi_{j}^{ \pm}\left(\vec{r}_{T}, t\right)=0, \quad j=i, f,
$$

where $H_{i}=K_{e l}+V_{T}+V_{0}(t)$ and $H_{f}=K_{e l}+V_{0}(t)$ are the initial and final unperturbed Hamiltonians, respectively.

In the final channel, to solve Eq. (4) we approximate $V_{0}(t)$ around the $P$ position; that is,

$$
V_{0}\left(\vec{r}_{T}, t\right) \simeq V_{0}\left(\vec{R}_{T}(t), t\right)-\vec{E}_{0}\left(\vec{R}_{T}(t), t\right) \cdot\left[\vec{r}_{T}-\vec{R}_{T}(t)\right]
$$

where $\vec{E}_{0}\left(\vec{r}_{T}, t\right)=-\vec{\nabla}_{\vec{r}_{T}} V_{0}\left(\vec{r}_{T}, t\right)$ denotes the external field acting on the electron at the time $t$. Replacing Eq. (5) in Eq. (4), the solution for the final collision state is a Volkov wave function [19]

$$
\begin{aligned}
\phi_{f}^{-}\left(\vec{r}_{T}, t\right)= & (2 \pi)^{-3 / 2} \exp \left\{i\left[\vec{k}_{T}-\vec{A}_{f}(t)\right] \cdot \vec{r}_{T}\right\} \\
& \times \exp \left[-i B_{f}\left(\vec{k}_{T}, t\right)-i \varepsilon_{T} t\right]
\end{aligned}
$$

where $\vec{k}_{T}$ is the momentum of the ejected electron with respect to $T$ in absence of the external potential, and $\varepsilon_{T}$ $=k_{T}^{2} / 2$ is its energy. The vector potential of the external field in the final channel reads

$$
\vec{A}_{f}(t)=\vec{A}_{f}\left(t_{f}\right)-\int_{t_{f}}^{t} d t^{\prime} \vec{E}_{0}\left(\vec{R}_{T}\left(t^{\prime}\right), t^{\prime}\right),
$$

with $\vec{A}_{f}\left(t_{f}\right)$ being an arbitrary constant value, and

$$
\begin{aligned}
B_{f}(\vec{k}, t)= & \int_{t_{f}}^{t} d t^{\prime}\left[A_{f}^{2}\left(t^{\prime}\right) / 2-\vec{k} \cdot \vec{A}_{f}\left(t^{\prime}\right)+V_{0}\left(\vec{R}_{T}\left(t^{\prime}\right), t^{\prime}\right)\right. \\
& \left.+\vec{E}_{0}\left(\vec{R}_{T}\left(t^{\prime}\right), t^{\prime}\right) \cdot \vec{R}_{T}\left(t^{\prime}\right)\right],
\end{aligned}
$$

where $t_{f}=+\infty$.

In the same way, approximating $V_{0}(t)$ around the $T$ position in the initial channel, the initial collision state can be described by employing the so-called Coulomb-Volkov anzatz [20]

$$
\phi_{i}^{+}\left(\vec{r}_{T}, t\right)=\varphi_{i}\left(\vec{r}_{T}\right) \exp \left[-i \vec{A}_{i}(t) \cdot \vec{r}_{T}-i B_{i}(\overrightarrow{0}, t)-i \varepsilon_{i} t\right],
$$

where $\varphi_{i}$ and $\varepsilon_{i}$ are the wave function and energy corresponding to the initial state of the isolated target. The functions $\vec{A}_{i}$ and $B_{i}$ have similar forms to Eqs. (7) and (8), respectively, with the subindex $f$ replaced by the subindex $i$, the position $\vec{R}_{T}\left(t^{\prime}\right)$ replaced by $\overrightarrow{0}$ (the target position), and $t_{i}$ $=-\infty$.

Starting from the collision states given by Eqs. (6) and (9), it is possible to derive a generalization of the CDW-EIS approximation to describe the ionization process in the presence of an external potential. The proposed model will be here named as field distorted wave (FDW) approximation, because it essentially depends on the external field. As usual, the distorted wave functions are defined as [21]

$$
\chi_{i}^{+}\left(\vec{r}_{T}, t\right)=\phi_{i}^{+}\left(\vec{r}_{T}, t\right) E_{P}^{+}\left(-\vec{v}, \vec{r}_{P}\right)
$$

in the initial channel, and

$$
\chi_{f}^{-}\left(\vec{r}_{T}, t\right)=\phi_{f}^{-}\left(\vec{r}_{T}, t\right) D_{T}^{-}\left(\vec{k}_{T}, \vec{r}_{T}\right) D_{P}^{-}\left(\vec{k}_{P}, \vec{r}_{P}\right)
$$

in the final channel, where $\vec{k}_{P}=\vec{k}_{T}-\vec{v}$ is the final electron momentum with respect to $P$ in absence of $V_{0}, D_{c}^{ \pm}(\vec{k}, \vec{r})$ $=F_{c}^{ \pm}(k){ }_{1} F_{1}\left( \pm i Z_{c} / k, 1, \pm i k r-i \vec{k} \cdot \vec{r}\right)$ is the Coulomb distortion, and $E_{c}^{ \pm}(\vec{k}, \vec{r})=\exp \left[\mp i Z_{c} / k \ln (k r \mp \vec{k} \cdot \vec{r})\right]$ is the eikonal phase, with $c=P, T$. In the definition of $D_{c}^{ \pm}$, the function ${ }_{1} F_{1}$ denotes the confluent hypergeometric function, $k=|\vec{k}|$, and

$$
F_{c}^{ \pm}(k)=\exp \left(\frac{\pi Z_{c}}{2 k}\right) \Gamma\left(1 \mp i Z_{c} / k\right), \quad c=P, T
$$

is a normalization factor (namely the Coulomb Jost function) that coincides with the value of the Coulomb wave function at $\vec{r}=\overrightarrow{0}$, with $\Gamma$ being the gamma function. 
In the first order of a distorted-wave theory, the transition amplitude as a function of the impact parameter $\vec{\rho}$ reads [22]

$$
\mathcal{A}_{i f}^{\mathrm{FDW}}(\vec{\rho})=-i \int_{-\infty}^{+\infty} d t\left\langle\chi_{f}^{-}(t)\left|H_{e l}-i \frac{\partial}{\partial t}\right| \chi_{i}^{+}(t)\right\rangle .
$$

The transition matrix can be derived from Eq. (13) by using the eikonal transformation, $T_{i f}^{\mathrm{FDW}}$ $=v(2 \pi)^{-3} \int d \vec{\rho} \mathcal{A}_{i f}^{\mathrm{FDW}}(\vec{\rho}) \exp (-i \vec{\eta} \cdot \vec{\rho})$, where $\vec{\eta}$ is the component of the transferred momentum perpendicular to $\vec{v}$. After simple algebra and changing variables, the $T$-matrix element reads

$$
\begin{aligned}
T_{i f}^{\mathrm{FDW}}= & \frac{1}{(2 \pi)^{9 / 2}} \int d \vec{r}_{T} \int d \vec{r}_{P} \varphi_{i}\left(\vec{r}_{T}\right) E_{P}^{+} \\
& \times \exp \left[-i \vec{Q}_{P} \cdot \vec{r}_{P}-i \vec{Q}_{A}(\tau) \cdot \vec{r}_{T}+i B(\tau)\right] \\
& \times\left\{-\vec{A}_{f}(\tau) \cdot\left[D_{P}^{-*} \vec{\nabla}_{\vec{r}} D_{T}^{-*}+D_{T}^{-*} \vec{\nabla}_{r_{P}} D_{P}^{-*}\right]\right. \\
& \left.+i \vec{\nabla}_{\vec{r}_{T}} D_{T}^{-*} \cdot \vec{\nabla}_{\vec{r}_{P}} D_{P}^{-*}\right\},
\end{aligned}
$$

where $\vec{Q}_{P}=-\vec{\eta}-\hat{v}\left(\varepsilon_{i}-\varepsilon_{T}\right) / v$ is the projectile transferred momentum, $\vec{Q}_{A}(\tau)=\vec{k}_{T}-\vec{A}(\tau)-\vec{Q}_{P}$,

$$
\begin{aligned}
& \vec{A}(\tau)=\vec{A}_{f}(\tau)-\vec{A}_{i}(\tau), \\
& B(\tau)=B_{f}\left(\vec{k}_{T}, \tau\right)-B_{i}(\overrightarrow{0}, \tau),
\end{aligned}
$$

and $\tau=\hat{v} \cdot\left(\vec{r}_{T}-\vec{r}_{P}\right) / v$, with $\hat{v}=\vec{v} / v$. To simplify notation, in Eq. (14) we have denoted $E_{P}^{+} \equiv E_{P}^{+}\left(-\vec{v}, \vec{r}_{P}\right), \quad D_{T}^{-}$ $\equiv D_{T}^{-}\left(\vec{k}_{T}, \vec{r}_{T}\right)$, and $D_{P}^{-} \equiv D_{P}^{-}\left(\vec{k}_{P}, \vec{r}_{P}\right)$. Note that on right hand of Eq. (14), the independent coordinates $\vec{r}_{T}$ and $\vec{r}_{P}$ are mixed as a consequence of the parameter $\tau$ that includes both; therefore, integrals on $\vec{r}_{T}$ and $\vec{r}_{P}$ cannot be calculated separately unless an approximation over $\tau$ has been done.

\section{B. Electron emission from surfaces}

The FDW approximation is here applied to study the electron emission in grazing-ion-surface collisions. When the incident ion approaches the surface, it induces the ejection of electrons from target atoms located at the topmost atomic layer, and this atomic ionization process is developed in the presence of the potential $V_{0}$ originated by the surface. In the case of metal surfaces, $V_{0}$ represents the surface induced potential, while for insulators it essentially corresponds to the track surface potential.

Due to the geometry of the problem, it is convenient to change the reference frame by one fixed to the position of the first atomic layer, with the projectile trajectory contained in the $x-z$ plane, and the surface in the $x-y$ plane (see Fig. 1). In the new coordinate system the external potential $V_{0}$ is expressed as $V_{0}(\vec{r}, t)$, where $\vec{r}$ is the position vector of $e$ in the surface reference frame, and the time dependence is deter-

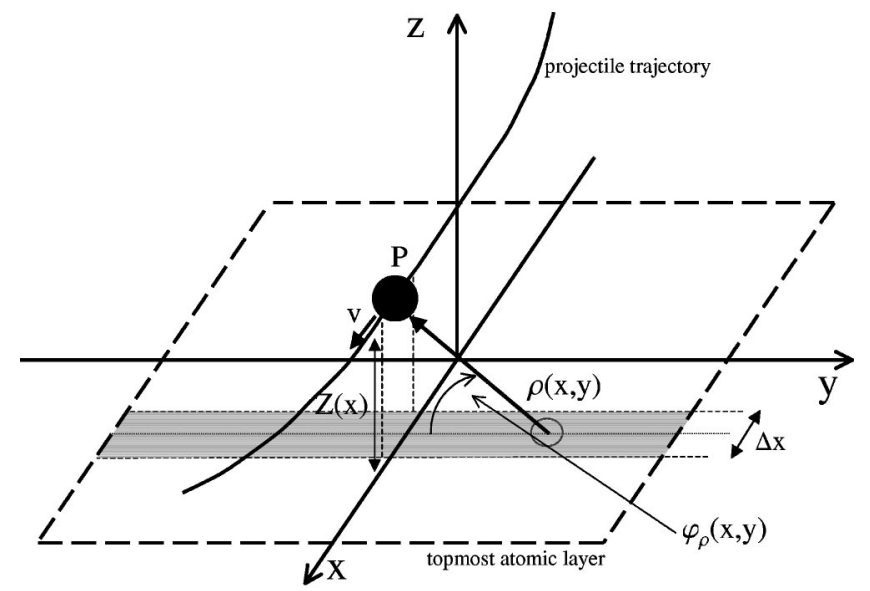

FIG. 1. Schematic picture of the coordinate system.

mined by the projectile position $\vec{R}(t)$.

In grazing scattering, the projectile trajectory can be divided into differential portions, with width $\Delta x$, situated at different distances $Z(x)$ from the surface. In every portion the component of the ion velocity perpendicular to the surface $v_{z}$ is considered negligible, and the projectile moving parallel to the surface with velocity $\vec{v}_{s}=\left(v_{s}, 0,0\right)$ ionizes the target atoms located in the corresponding surface band $\Delta x$. This implies that for a given surface atom placed at $\vec{x}$ $=(x, y, 0)$, the whole atomic collision process occurs while the projectile is moving along a portion $\Delta x$ of its trajectory. Then, as the external field does not vary appreciably on the $\Delta x$ interval, the vector $\vec{R}(t)$ can be taken as a constant, i.e. $\vec{R}(t) \simeq \vec{R}(x) \equiv(x, 0, Z(x))$, as far as the surface potential is concerned. According to this assumption and changing the variable $t^{\prime}$ by $x^{\prime}=v_{s} t^{\prime}$ in Eq. (7), the initial and final vector potentials involved in the ionization process read

$$
\vec{A}_{j}(x)=-\left(\frac{d_{s}}{2}\right) \frac{\vec{E}_{0}\left(\vec{r}_{j}, x\right)}{v_{s}}, \quad j=i, f
$$

where $x=v_{s} t$ is the position of the projectile at the time $t$, when the collision with the surface atom situated at $\vec{x}$ $=(x, y, 0)$ takes place. The positions around which the surface field is evaluated are $\vec{r}_{i}=\vec{x}$ and $\vec{r}_{f}=\vec{R}(x)$ in the initial and final channels, respectively, and $d_{s}=\Delta x$ denotes the distance between surface atoms. In Eq. (17), we have fixed the zero of the vector potential at the beginning of the atomic collision, i.e., $\vec{A}_{j}\left(x-d_{s} / 2\right)=0$ for $j=i, f$. Moreover, the parameter $\tau$ can be expressed as $\tau=\hat{v}_{s} \cdot \vec{R} / v_{s} \simeq x / v_{s}$, which becomes independent of the electron position, allowing us to uncouple the integrals on $\vec{r}_{T}$ and $\vec{r}_{P}$ in Eq. (14).

Taking into account that the electron is ejected from the surface with an effective final momentum $\vec{k}_{f}=\vec{k}_{T}-\vec{A}_{f}(x)$, as observed from Eq. (6), the transition matrix reduces to 


$$
\begin{aligned}
T_{i f}^{\mathrm{FDW}}= & \frac{\exp [i B(x)]}{(2 \pi)^{3 / 2}} \\
& \times\left\{i \vec{N}_{i}^{T-*}\left(\vec{k}_{T},-\vec{Q}_{T}(x)\right) \cdot \vec{T}_{P}^{(--,+)}\left(\vec{k}_{P},-\vec{v}_{s}, \vec{Q}_{P}\right)\right. \\
& -\vec{A}_{f}(x) \cdot\left[\vec{N}_{i}^{T-*}\left(\vec{k}_{T},-\vec{Q}_{T}(x)\right) U_{P}^{(-,+)}\left(\vec{k}_{P},-\vec{v}_{s}, \vec{Q}_{P}\right)\right. \\
& \left.\left.+L_{i}^{T-*}\left(\vec{k}_{T},-\vec{Q}_{T}(x)\right) \vec{T}_{P}^{(-,+)}\left(\vec{k}_{P},-\vec{v}_{s}, \vec{Q}_{P}\right)\right]\right\}, \quad(18)
\end{aligned}
$$

where $\vec{k}_{T}=\vec{k}_{f}+\vec{A}_{f}(x)$ and $\vec{k}_{P}=\vec{k}_{f}+\vec{A}_{f}(x)-\vec{v}_{s}$ are the electron momenta with respect to $T$ an $P$, respectively, involved in the hard atomic collision, and $\vec{Q}_{T}(x)=\vec{k}_{f}-\vec{Q}_{P}+\vec{A}_{i}(x)$. The function $B(x)$ is obtained by replacing $\tau$ by $x / v_{s}$ in Eq. (16), and the auxiliary functions are Nordsieck-type integrals:

$$
\begin{aligned}
{\left[\begin{array}{c}
L_{i}^{T-}\left(\vec{k}_{1}, \vec{q}\right) \\
\vec{N}_{i}^{T-}\left(\vec{k}_{1}, \vec{q}\right)
\end{array}\right]=} & \int \frac{d \vec{r}}{(2 \pi)^{3 / 2}}\left[\begin{array}{c}
1 \\
\vec{\nabla}_{r}
\end{array}\right] D_{T}^{-}\left(\vec{k}_{1}, \vec{r}\right) \\
& \times \exp (-i \vec{q} \cdot \vec{r}) \varphi_{i}^{*}(\vec{r})
\end{aligned}
$$

and

$$
\begin{aligned}
{\left[\begin{array}{c}
U_{P}^{(-,+)}\left(\vec{k}_{1}, \vec{k}_{2}, \vec{q}\right) \\
\vec{T}_{P}^{(-,+)}\left(\vec{k}_{1}, \vec{k}_{2}, \vec{q}\right)
\end{array}\right]=} & \int \frac{d \vec{r}}{(2 \pi)^{3 / 2}}\left[\begin{array}{c}
1 \\
\vec{\nabla}_{\vec{r}}
\end{array}\right] D_{P}^{-*}\left(\vec{k}_{1}, \vec{r}\right) \\
& \times \exp (-i \vec{q} \cdot \vec{r}) E_{P}^{+}\left(\vec{k}_{2}, \vec{r}\right),
\end{aligned}
$$

which have closed forms [23]. In Eq. (18), the exponential factor depending on $B(x)$ is a fixed phase factor, which does not affect the ionization probability.

Even though Eq. (18) can be exactly evaluated, with the aim of simplifying the calculations, we have introduced two additional approximations. (i) As electrons initially bound to the surface atoms are weakly affected by the action of the surface potential, we dropped the initial vector potential $\vec{A}_{i}$ that modified the unperturbed atomic state $\varphi_{i}$ as given in Eq. (9). (ii) Since the perturbation introduced by the surface potential $V_{0}$ is much smaller than the Coulomb interaction with the projectile, we have neglected its first-order contribution to the $T$-matrix element, keeping only the first term of Eq. (18). Then, the $T$-matrix element reduces, except for an unimportant phase factor, to

$$
T_{i f}^{\mathrm{FDW}} \simeq(2 \pi)^{-3 / 2} \vec{N}_{i}^{T-*}\left(\vec{k}_{T},-\vec{Q}\right) \cdot \vec{T}_{P}^{(-,+)}\left(\vec{k}_{P},-\vec{v}_{s}, \vec{Q}_{P}\right),
$$

where $\vec{k}_{T}=\vec{k}_{f}+\vec{A}_{f}(x), \quad \vec{k}_{P}=\vec{k}_{f}+\vec{A}_{f}(x)-\vec{v}_{s}$, and $\vec{Q}=\vec{k}_{f}$ $-\vec{Q}_{P}$ is the usual definition of the transferred momentum. Therefore, in the FDW formalism the action of the external field on the ejected electron produces a supplementary momentum transfer $\vec{A}_{f}(x)$, which depends on the point of the projectile trajectory considered. If the vector potential $\vec{A}_{f}$ was fixed equal to zero, Eq. (21) would become equivalent to the $T$-matrix obtained with the CDW-EIS approximation for ion-atom collisions [21].
Finally, the atomic ionization probability depending on $\vec{\rho}$, $P_{i f}^{\mathrm{FDW}}(\vec{\rho})=\left|\mathcal{A}_{i f}^{\mathrm{FDW}}(\vec{\rho})\right|^{2}$, is derived from Eq. (21) by using again the eikonal transformation

$$
\mathcal{A}_{i f}^{\mathrm{FDW}}(\vec{\rho})=\frac{2 \pi}{v_{s}} \int d \vec{\eta} T_{i f}^{\mathrm{FDW}} \exp (i \vec{\eta} \cdot \vec{\rho}) .
$$

The differential probability of electron emission from the surface, $d P_{i} / d \vec{k}_{f}$, corresponding to the transition from the initial state $i$ to the final state with momentum $\vec{k}_{f}$ and energy $\varepsilon_{f}=k_{f}^{2} / 2$, is obtained integrating $P_{i f}^{\mathrm{FDW}}$ on both the surface band $\Delta x$ and on the projectile trajectory. It reads [18]

$$
d P_{i} / d \vec{k}_{f}=\delta_{S} \int_{-\infty}^{+\infty} d x \int_{-\infty}^{+\infty} d y P_{i f}^{\mathrm{FDW}}(\vec{\rho}(x, y))
$$

where $\vec{\rho}$ depends on the position of the surface atom considered,

$$
\rho(x, y)=\sqrt{y^{2}+Z^{2}(x)}, \quad \varphi_{\rho}(x, y)=\arctan \left(\frac{Z(x)}{y}\right)
$$

being the modulus and the azimuthal angle, respectively, of $\vec{\rho}(x, y), Z(x)$ denotes the classical trajectory of the projectile, and $\delta_{S}$ is surface atomic density, which is considered as a constant.

\section{RESULTS}

As a first benchmark for the proposed formalism, we consider the collision system composed by $100 \mathrm{keV}$ protons grazingly impinging on a solid surface. Since the energy shift of the CEP strongly depends on the electronic properties of the surface, two different types of solids are examined: $\mathrm{LiF}(100)$ and $\mathrm{Al}(111)$, insulator and metal surfaces, respectively. For both cases, experimental data of the energy shift of the CEP are available [7,5].

The same way as in theories for ion-atom collisions, in the FDW model the position of the CEP is related to threshold laws characteristics of the final $P-e$ interaction. At every position $\vec{R}(x)$ of the projectile, the behavior of the atomic probability $P_{i f}^{\mathrm{FDW}}$ near the break-up threshold is determined by the normalization Coulomb factor $F_{P}^{-}\left(k_{P}\right)$, defined by Eq. (12), which is contained in the function $\vec{T}_{P}^{(-,+)}$in Eq. (21). This Coulomb factor displays a cusp shape as $k_{P} \rightarrow 0$, that is, for values of the final electron momentum $\vec{k}_{f}$ close to $\vec{v}_{s}-\vec{A}_{f}(x)$. Then, in the proposed model the peak position in the electron spectrum is displaced by an additional transferred momentum $\vec{A}_{f}$, which is proportional to surface interaction at the projectile position, as proposed earlier by Burgdörfer [24]. In the evaluation of the probability of electron emission from the surface, this Coulomb peak is convoluted along the ion trajectory, giving rise to a small broadening.

As we are interested in studying the energy shift of the CEP, we have first evaluated the transition matrix from Eq. (21) without including the momentum $\vec{A}_{f}$ (CDW-EIS approximation). From such a value we derive an approximated 
result for $T_{i f}^{\mathrm{FDW}}$ by replacing the Coulomb factors $F_{T}^{-}\left(k_{f}\right)$ and $F_{P}^{-}\left(\left|\vec{k}_{f}-\vec{v}_{s}\right|\right)$, involved in the usual CDW-EIS transition matrix, by the new shifted Coulomb factors $F_{T}^{-}\left(k_{T}\right)$ and $F_{P}^{-}\left(k_{P}\right)$ [with $\vec{k}_{T}=\vec{k}_{f}+\vec{A}_{f}(x)$ and $\vec{k}_{P}=\vec{k}_{f}+\vec{A}_{f}(x)-\vec{v}_{s}$ ], contained in the auxiliary functions $\vec{N}_{i}^{T-}$ and $\vec{T}_{P}^{(-,+)}$, respectively, in Eq. (21). Besides, as the CDW-EIS approximation displays asymmetric behavior around the CEP, a factor that averages this asymmetry is used to derive the FDW transition matrix from the CDW-EIS one.

For every initial state, the evaluation of $d P_{i} / d \vec{k}_{f}$ involves a double integral over the transferred momentum $\vec{\eta}$, as given by Eq. (22), and other two integrals on the surface plane $(x, y)$, as given by Eq. (23). The integration on the variables $\vec{\eta}$ and $y$ was numerically evaluated with a relative error lower than $3 \%$, while the further integration on the variable $x$ was solved by interpolating approximately 20 pivots on the classical trajectory $Z(x)$.

\section{A. Insulator surfaces}

In this section we concentrate on $\mathrm{H}^{+}$impinging on an $\mathrm{LiF}(100)$ surface with $100 \mathrm{keV}$ energy and the angle of incidence $\theta_{i}=0.7 \mathrm{deg}$. The LiF can be considered as the typical example of insulator: it has a narrow valence band, a large work function, and a wide band gap. Furthermore, as valence electrons are localized around ionic centers (ionic crystal), we can consider that the electron emission is principally caused by the ionization of electrons bounded to target ions. At the considered energy, the ionization of the ground state of $\mathrm{Li}^{+}$is small, and most of the ejected electrons come from localized states around the $\mathrm{F}^{-}$ions. We employ Hartree-Fock wave functions for negative ions [25] to represent the bound states of the $\mathrm{F}^{-}$ion, and no correction is included to take into account that target ions are part of a surface. Since at $100 \mathrm{keV}$ impact energy the contribution of the $K$ shell of $\mathrm{F}^{-}$ is negligible, only the $L$ shell is considered in our calculations.

\section{Determination of the vector potential $\vec{A}_{f}$}

As a consequence of the low conductivity of the medium, the ionization of $\mathrm{F}^{-}$ions along the projectile path originates a surface charge density which is responsible for the track potential. When the projectile is at $\vec{R}(x)$ position, the track field acting on the electron placed at the $\vec{r}$ position reads

$$
\begin{aligned}
\vec{E}_{0}(\vec{r}, x)= & q_{e} \delta_{S}^{\left(F^{-}\right)} \int_{-\infty}^{x} d x^{\prime} \int_{-\infty}^{+\infty} d y^{\prime} \\
& \times P_{\text {ionz }}\left(\vec{\rho}\left(x^{\prime}, y^{\prime}\right)\right) \frac{\left(\vec{r}-\vec{x}^{\prime}\right)}{\left|\vec{r}-\vec{x}^{\prime}\right|^{3 / 2}},
\end{aligned}
$$

where $\vec{x}^{\prime}=\left(x^{\prime}, y^{\prime}, 0\right)$ denotes the position of the $\mathrm{F}^{-}$ion involved in the ionization process, and $P_{\text {ionz }}(\vec{\rho})$ is the total ionization probability of $\mathrm{F}^{-}$as a function of the impact parameter $\vec{\rho}\left(x^{\prime}, y^{\prime}\right)$, which is defined in terms of the classical trajectory $Z\left(x^{\prime}\right)$ as given by Eq. (24). The factor $\delta_{S}^{\left(F^{-}\right)}$is the surface density of $\mathrm{F}^{-}$ions, and $q_{e}=-1$ is the electron charge.

For insulators, the track potential is expected to represent the dominant surface interaction that affects the movement of ejected electrons [7]. As a first estimate, we neglect the contribution of the induced surface potential to $V_{0}$, assuming that the dynamic response of the medium is weak in comparison with the track interaction $[26,27]$. The field $\vec{E}_{0}$, given by Eq. (25), attracts the emitted electron towards the surface with an intensity that depends on the position of the projectile, being different on the incoming and outgoing projectile paths. To evaluate the ionization probability $P_{\text {ionz }}(\vec{\rho})$ involved in Eq. (25) we employ the CDW-EIS approximation [21], using an effective charge that satisfies the initial binding energy to describe the $T$-e Coulomb interaction. As the band gap is not expected to be significant at this high impact energy $[28,29]$, it was not considered in the calculation. The classical trajectory of the projectile was obtained with the Molière potential [30], adding $\mathrm{Li}$ and $\mathrm{F}$ contributions. From the track field [28] we derive the vector potential $\vec{A}_{f}$, as given by Eq. (17), with $d_{s} \simeq 7.6$ a.u. the separation between $\mathrm{F}^{-}$ions on the topmost atomic layer. Components parallel $\left(A_{f x}\right)$ and perpendicular $\left(A_{f z}\right)$ to the surface of $\vec{A}_{f}$ are shown in Fig. 2 as a function of the projectile distance to the atomic surface $Z(x)$, for the incoming and outgoing trajectories. Results obtained along both paths are quite similar: $A_{f x}$ and $A_{f z}$ components decrease almost linearly, in logarithmic scale, as the distance to the surface increases. And near the surface, for $Z(x) \lesssim 2.0$ a.u., the component parallel to the surface prevails against the perpendicular one, i.e. $A_{f x}>A_{f z}$, while the opposite happens for long distances, where $A_{f x}<A_{f z}$.

\section{FDW Jost function}

In order to test the changes introduced by the vector potential $\vec{A}_{f}$, we have studied the behavior of the FDW Jost function corresponding to the Coulomb projectile distortion in the final channel,

$$
J_{P}^{\mathrm{FDW}}\left(\vec{k}_{f}\right)=\left|F_{P}^{-}\left(k_{P}\right)\right|^{2},
$$

with $\vec{k}_{P}=\vec{k}_{f}+\vec{A}_{f}(x)-\vec{v}_{s}$, which dominates the energy spectrum obtained with the FDW approximation in the region of convoy-electron emission. The function $J_{P}^{\mathrm{FDW}}$ for electrons ejected at the specular-reflection direction is plotted in Fig. 3 as a function of the electron energy $\varepsilon_{f}$, considering the proton at the closest distance to the surface, i.e. $\vec{R}(0)$ $=(0,0, Z(0))$, with $Z(0)=0.29$ a.u. At this position, the value of the vector potential is $\vec{A}_{f}(0)=(0.22,0,0.11)$ a.u. The Jost function in absence of the track potential $J_{P}\left(\vec{k}_{f}\right)$ $=\left|F_{P}^{-}\left(\left|\vec{k}_{f}-\vec{v}_{s}\right|\right)\right|^{2}$, which is involved in the CDW-EIS approximation, is also displayed as a comparison. From Fig. 3, the maximum of the function $J_{P}^{\mathrm{FDW}}$, which is associated with CEP, is not only energy displaced but also lowered as a consequence of the momentum transfer $\vec{A}_{f}(0)$. This is because the Coulomb divergency as $\vec{k}_{P} \rightarrow \overrightarrow{0}$ is now centered at the 


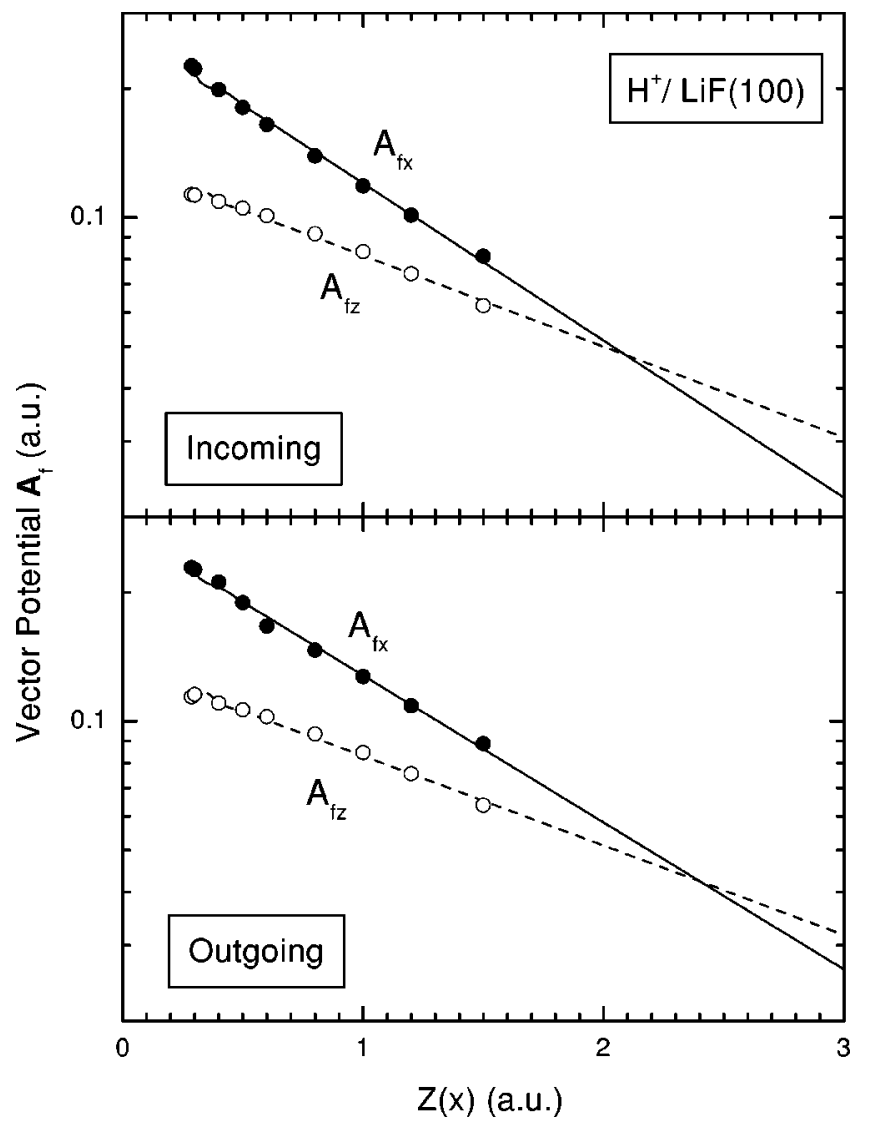

FIG. 2. Vector potential $\vec{A}_{f}$ of the track field, as given by Eq. (17), for $100 \mathrm{keV}$ protons impinging on a $\mathrm{LiF}(100)$ surface. The variable $Z(x)$ denotes the projectile distance to the topmost atomic layer, in the incoming $(x<0)$ and outgoing $(x>0)$ paths. Solid dots (solid line), component of $\vec{A}_{f}$ parallel to the surface, $A_{f x}$; and open dots (dashed line), component of $\vec{A}_{f}$ perpendicular to the surface, $A_{f z}$. Lines represent the used interpolation.

position $\vec{k}_{f}^{(P)}=\vec{v}_{s}-\vec{A}_{f}(0)$ in the momentum space, which corresponds to electrons emitted inside the solid (with $k_{f z}^{(P)}$ $\left.=-A_{f z}(0)<0\right)$, not observed in experiments. Then, in the FDW approximation the structure corresponding to the CEP is wider than that of ion-atom scattering. The FDW curve displayed in Fig. 3 only corresponds to the contribution of the position $\vec{R}(0)$, and the shape of the total spectrum is obtained by integrating the ionization probability along the trajectory of the ion, which broadens the peak even more.

\section{Electron distribution}

The double differential probability of electron emission $d^{2} P / d \varepsilon_{f} d \Omega_{f}=k_{f} \Sigma_{i} d P_{i} / d \vec{k}_{f}$, calculated from Eq. (23) by adding the contributions of $2 s$ and $2 p$ states of $\mathrm{F}^{-}$, is plotted in Fig. 4 as a function of the electron energy $\varepsilon_{f}$. The solid angle $\Omega_{f}$ coincides with the direction of the outgoing projectile (the electron emission angle is $\theta_{f}=0.7 \mathrm{deg}$, measured with respect to the surface and on the scattering plane). In Fig. 4, results from the FDW model are compared with those obtained by considering the track potential equal to zero, i.e. $\vec{A}_{f}=0$. The FDW energy distribution displays a structure



FIG. 3. Jost function involved in the calculation of the emission probability, as a function of the electron energy, for $100 \mathrm{keV}$ protons colliding with a $\mathrm{LiF}(100)$ surface with the incidence angle $\theta_{i}=0.7^{\circ}$. The electron observation angle is $\theta_{f}=0.7^{\circ}$, and the projectile is considered at the closest distance to the surface, $Z(0)$. Solid line, Jost function $J_{P}^{\mathrm{FDW}}\left(\vec{k}_{f}\right)$, as defined by Eq. (26), which is contained in the FDW approximation; and dotted line, Jost function in the absence of the surface interaction, $J_{P}\left(\vec{k}_{f}\right)=\mid F_{P}^{-}\left(\mid \vec{k}_{f}\right.$ $\left.-\vec{v}_{s} \mid\right)\left.\right|^{2}$. A schematic drawing of the position of the CEP in the momentum space is shown in the inset.

around the CEP that is markedly broadened and shifted towards a lower energy. And the energy shift $\Delta \varepsilon^{(C E)}$, defined as the difference between the positions of the maximum with and without including $\vec{A}_{f}$, is $\Delta \varepsilon^{(C E)} \simeq-4 \mathrm{eV}$, which is close to the experimental value $\Delta \varepsilon_{\text {expt }}^{(C E)} \simeq-5 \mathrm{eV}$ [7]. Fur-



FIG. 4. Double differential probability of electron emission $d^{2} P / d \varepsilon_{f} d \Omega_{f}$ for $100 \mathrm{keV}$ protons impinging on a $\operatorname{LiF}(100)$ surface with the incidence angle $\theta_{i}=0.7^{\circ}$. The electron ejection angle is $\theta_{f}=0.7^{\circ}$. Solid line, results obtained with the FDW approximation; and dotted line, values from the CDW-EIS approximation without including the surface interaction. 


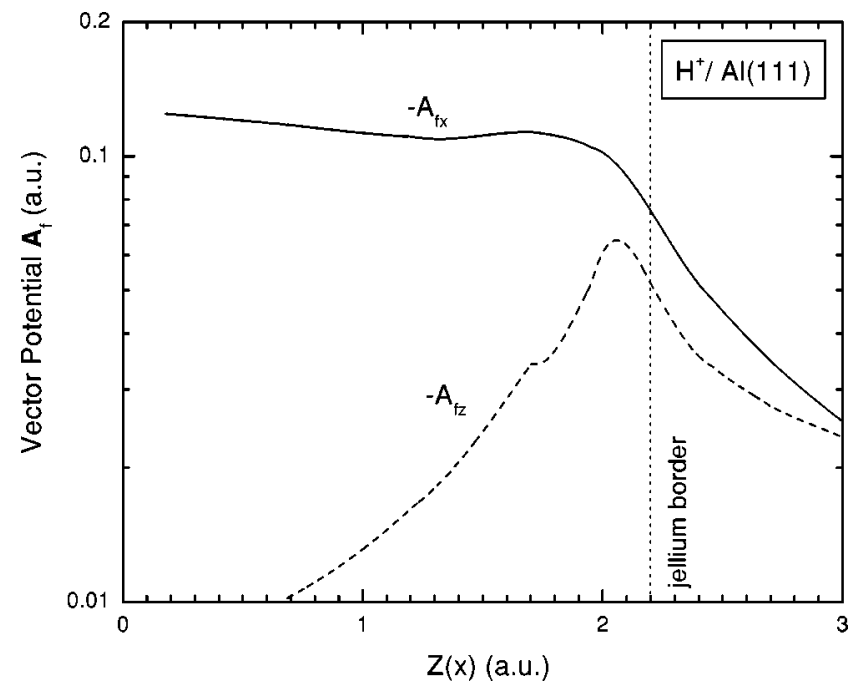

FIG. 5. Vector potential $\vec{A}_{f}$ of the induced surface field, derived from Eq. (17), for $100 \mathrm{keV}$ protons impinging on an $\mathrm{Al}(111)$ surface. The variable $Z(x)$ denotes the projectile distance to the topmost atomic layer. Solid and dashed lines, absolute values of the components of $\vec{A}_{f}$ parallel, $A_{f x}$, and perpendicular, $A_{f z}$, respectively, to the surface.

thermore, the shape of the FDW electron distribution qualitatively agrees with experimental spectra obtained for slightly lower impact energies [7]. Therefore, the proposed model seems to give an appropriate description of the broadening and the energy shift of the CEP, which are the footprints of the presence of the track potential. Note that other effects not included in our calculations, such as screening by low-energy electrons or polarization of the surrounding anions, might reduce the strength of the track potential.

\section{B. Metal surfaces}

Our study on metal surfaces is confined to $100 \mathrm{keV}$ protons colliding on an $\mathrm{Al}(111)$ surface with the angle of incidence $\theta_{i}=1 \mathrm{deg}$. The parameters used to describe the aluminum surface are the following: The interplanar separation is $d=4.4$ a.u., the Fermi energy is $E_{F}=0.414$ a.u. (the Fermi velocity $v_{F}=0.91$ a.u.), the work function is $\varepsilon_{W}$ $=0.15$ a.u., the surface plasma frequency is $w_{s}=0.4$ a.u., and the plasmon width is $\gamma=0.037$ a.u. [31]. The trajectory of the incident ion is determined by using the Molière potential [30] plus the dynamical image potential given in Ref. [31].

\section{Determination of the vector potential $\vec{A}_{f}$}

In metals the surface interaction $V_{0}$ is due to the induced surface potential, which is associated with the dielectric response of the material. We employ the specular-reflection (SR) model [32] to derive the induced field $\vec{E}_{0}$ acting on the electron at the projectile position [33]. The field $\vec{E}_{0}$ includes the image field induced by the projectile and the self-image field induced by the ejected electron, considering that both move together. In Fig. 5 we have plotted the vector potential

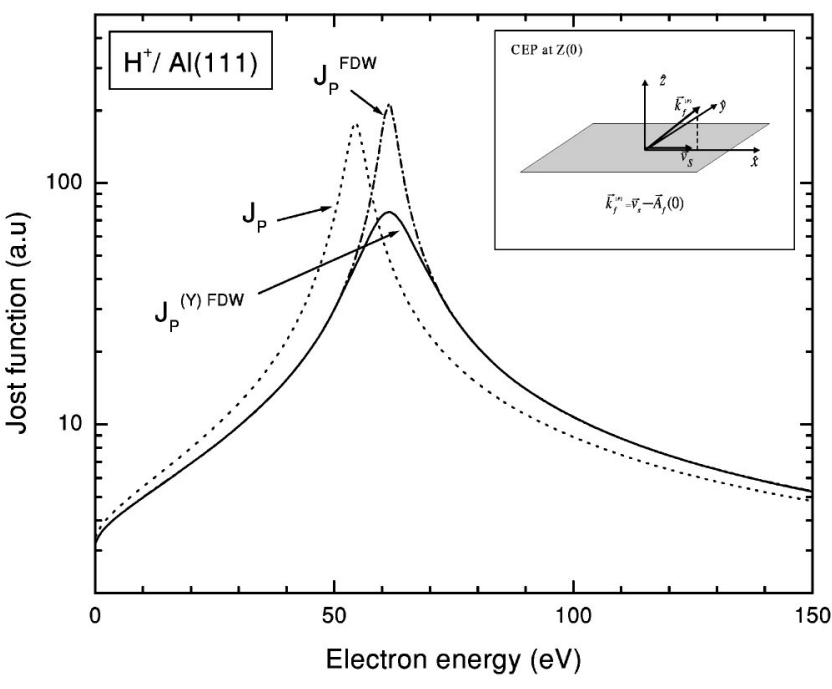

FIG. 6. Similar to Fig. 3 for $100 \mathrm{keV}$ protons colliding with an $\mathrm{Al}(111)$ surface with the incidence angle $\theta_{i}=1 \mathrm{deg}$. The electron emission angle is $\theta_{f}=1 \mathrm{deg}$. Solid line, Jost function $J_{P}^{(Y) \text { FDW }}\left(\vec{k}_{f}\right)$ corresponding to the Yukawa potential, with momentum $\vec{k}_{P}=\vec{k}_{f}$ $+\vec{A}_{f}(x)-\vec{v}_{s}$; dot-dashed line, Jost function $J_{P}^{\mathrm{FDW}}\left(\vec{k}_{f}\right)$, as defined by Eq. (26); and dotted line, Jost function in absence of the surface interaction, $J_{P}\left(\vec{k}_{f}\right)=\left|F_{P}^{-}\left(\left|\vec{k}_{f}-\vec{v}_{s}\right|\right)\right|^{2}$.

$\vec{A}_{f}$, obtained from Eq. (17) with $d_{s} \simeq 5.4$ a.u., as a function of the projectile distance to the atomic surface. The value $Z_{J}=2.2$ a.u corresponds to the position of the jellium border, which is displaced one half of the interplanar atomic distance from the topmost atomic layer. The vector potential $\vec{A}_{f}$ for metals differs not only in the sign from the one for insulators. Inside the solid, for $Z \leqslant Z_{J}$, the component parallel to the surface $A_{f x}$ is almost constant, while in the vacuum it rapidly decreases for increasing $Z$ distances. Instead, the perpendicular component $A_{f z}$ tends to zero inside and outside the solid, when the distance to the jellium border augments, and it displays a maximum near the jellium edge. At the position of maximum approach to the surface, $Z(0)=0.18$ a.u., the vector potential is $\vec{A}_{f}(0)=(-0.12,0,-0.008)$ a.u. Note that the absolute value of $A_{f x}(0)$ is the same order of that obtained for $\mathrm{LiF}$, while the component $A_{f z}(0)$ is one order of magnitude smaller.

\section{FDW Jost function}

As in the case of insulators, to analyze the effect of the vector potential $\vec{A}_{f}$ on the electron energy distribution, in Fig. 6 we plot the FDW Jost function $J_{P}^{\mathrm{FDW}}$, defined in Eq. (26), for electrons emitted with $\theta_{f}=1 \mathrm{deg}$ at the closest distance $Z(0)$. The Jost function $J_{P}$, corresponding to $\vec{A}_{f}=\overrightarrow{0}$, is also shown in Fig. 6 as a reference. For the Al surface, the maximum $J_{P}^{\mathrm{FDW}}$, which is related to the CEP, is displaced towards higher energies as a consequence of the acceleration caused by the induced potential. And the Coulomb divergency of the factor of $J_{P}^{\mathrm{FDW}}$ as $\vec{k}_{P} \rightarrow \overrightarrow{0}$ corresponds to electrons ejected almost parallel to the surface, with $\vec{k}_{f} \rightarrow \vec{k}_{f}^{(P)}$ 


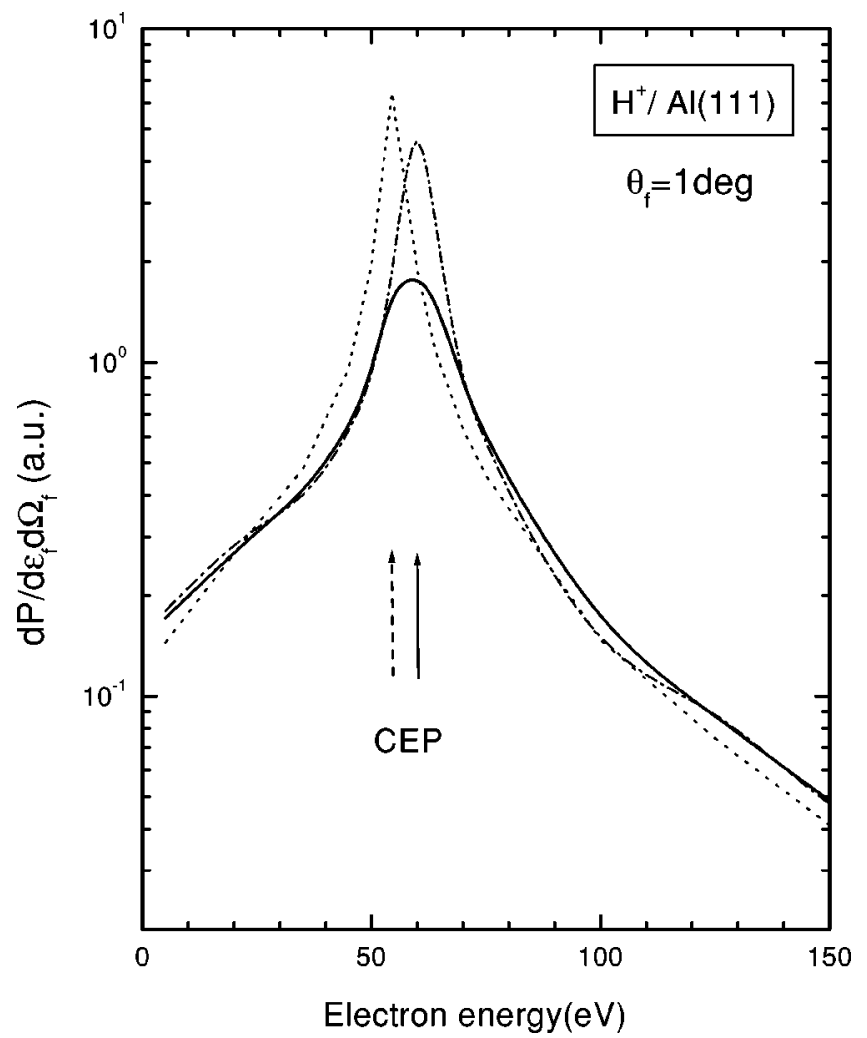

FIG. 7. Double differential probability of inner-shell emission $d^{2} P / d \varepsilon_{f} d \Omega_{f}$ for $100 \mathrm{keV}$ protons impinging on an $\mathrm{Al}(111)$ surface with the incidence angle $\theta_{i}=1 \mathrm{deg}$. The electron ejection angle is $\theta_{f}=1 \mathrm{deg}$. Solid (dot-dashed) line, results derived from the FDW approximation with (without) including the Yukawa Jost function; and dotted line, values from the CDW-EIS approximation in absence of the surface interaction.

$=\vec{v}_{s}-\vec{A}_{f}(0)$, being now $k_{f z}^{(P)}=-A_{f z}(0)>0$. The function $J_{P}^{\text {FDW }}$ displayed in Fig. 6 must be then integrated along the projectile path, giving rise to a small broadening of the Coulomb peak.

Although the FDW approximation provides an energy displacement of the CEP that seems to be adequate for both insulators and metals, in this last case the shape of the peak is almost as sharp as that obtained without including the surface interaction (see Fig. 7). This is because the model does not take into account that in collisions with metals the projectile penetrates into the jellium, and it is shielded by valence electrons, which form the free-electron gas. In the present work, we introduce the dynamic screening of the $P-e$ interaction by replacing the Coulomb Jost function $J_{P}^{\mathrm{FDW}}\left(\vec{k}_{f}\right)$ by the Jost function $J_{P}^{(Y) F D W}\left(\vec{k}_{f}\right)$ corresponding to a simple Yukawa potential, $V_{P}^{(Y)}=-Z_{P} \exp \left(-\lambda r_{P}\right) / r_{P}$, with $\lambda$ $=w_{s}\left(v_{s}^{2}+v_{F}^{2} / 3\right)^{-1 / 2}$. This value of $\lambda$ satisfies the proper limits for high and low velocities. The Yukawa Jost function, $J_{P}^{(Y) \text { FDW }}\left(\vec{k}_{f}\right)$, is defined as [34] the value at the origin of the square modulus of the eigenfunction of the Yukawa potential with momentum $\vec{k}_{P}=\vec{k}_{f}+\vec{A}_{f}(x)-\vec{v}_{s}$. We numerically evaluate the function $J_{P}^{(Y) \text { FDW }}$ by using the code of Ref. [35], and results are plotted in Fig. 6. The Yukawa Jost function does not display the cusp-shape characteristic of Coulomb interactions, and its value at the maximum is smaller than that of $J_{P}^{\mathrm{FDW}}$.

\section{Electron distribution}

We have calculated the probability of electron emission from the surface $d^{2} P / d \varepsilon_{f} d \Omega_{f}=k_{f} \sum_{i} d P_{i} / d \vec{k}_{f}$ by employing the FDW approximation with the Yukawa Jost function. As the $\mathrm{Al}^{0}$ contains three electrons in the outermost shell $n$ $=3$, we consider that the target atoms cede these external electrons to the free-electron gas, keeping the rest of the electrons in the inner shells. At the considered impact energy, the contribution coming from the $K$ shell can be neglected, and only the initial states corresponding to the $L$ shell of neutral aluminum are included. The atomic bound states are described by the Hartree-Fock double- $z$ functions [25], and an effective charge satisfying the binding energy is again used to represent the final continuum state around the target. Results for $d^{2} P / d \varepsilon_{f} d \Omega_{f}$ are shown in Fig. 7 as a function of the electron energy for the ejection angle $\theta_{f}=1 \mathrm{deg}$, which coincides with the specular-reflection direction of the projectile. In Fig. 7 we also show the electron distribution obtained without including the projectile screening, and that derived in absence of the induced potential (with $\vec{A}_{f}=0$ ). The energy shift of the CEP provided by the FDW model is $\Delta \varepsilon^{(C E)}$ $\simeq 6 \mathrm{eV}$, which is quite similar to the experimental value $\Delta \varepsilon_{\text {expt }}^{(C E)} \simeq 7.5 \mathrm{eV}[5]$. Taking into account that electron emission by binary collisions with the valence band is negligible around the direction of specular reflection [13], the electron distribution derived from the FDW model approximately describes the total contribution around the CEP [5]. Note that in the proposed model, the broadening of the CEP for metals is not a consequence of the additional momentum transfer $\vec{A}_{f}$, but the screening of the $P-e$ interaction, as explored by Burgdörfer [24].

Finally, we should remark that while in insulators the electrons can be supposed as being directly ejected to the vacuum, in metals the electrons ionized with $\theta_{f}=1 \mathrm{deg}$ travel a long distance through the jellium before being emitted to the vacuum. Then, the ejected electron loses energy due to multiple collisions in the outgoing path, and this effect has not been included in the model. On other hand, in the proposed formalism the action of the induced potential is considered up to a distance $d_{s} / 2$, as given by Eq. (17), and the interaction between the surface and the convoy electron in the large exit path has not been taken into account. Therefore, for metals, the differential probability derived from the FDW approximation with the Yukawa Jost function represents the primary electron distribution. Subsequent acceleration as well as energy loss of the emitted electron should be treated on equal footing to describe the experimental situation.

\section{CONCLUSIONS}

We have presented a distorted-wave formalism, here called FDW approximation, to deal with the atomic ionization process in the presence of a time-dependent potential. 
The model, which is based on the expansion of the external potential in terms of the effective field, is used to study the electron emission from bound states to surface atoms, originated by grazing-ion-surface scattering. We have focalized our attention on the description of the convoy electrons at glancing ejection angles. In collisions with surfaces, the CEP looks like a broad structure in the energy spectrum, whose maximum is displaced with respect to its position in ionatom collisions due to the surface interaction. In the FDW model the energy shift of the CEP is taken into account as an additional electron transferred momentum $\vec{A}_{f}$, which depends on the surface interaction at the different positions of the projectile.

We apply the FDW formalism to collisions of protons on $\mathrm{LiF}(100)$ and $\mathrm{Al}(111)$ surfaces, which are insulator and metal materials, respectively. In the case of metals, to include the screening of the projectile caused by valence electrons, we have introduced the Jost function corresponding to the shielded $P-e$ potential in the final electron wave function. With this modification, the FDW model gives a proper description of both, the energy shift and the broadening of the CEP for the different considered targets. And the energy displacements of the CEP obtained with the FDW approximation are in good agreement with recent experimental data. An exhaustive comparison with experiments for different grazing conditions and ejection angles would be necessary to confirm the validity of the proposed theory.

\section{ACKNOWLEDGMENTS}

We are grateful to A. Arnau and V.H. Ponce for fruitful discussions. This work was supported in part by the ANPCyT (Projects No. PICT 03-03579 and No. PICT0306249) and UBACyT (Project No. 01-X044).
[1] K. Kimura, M. Tsuji, and M. Mannami, Phys. Rev. A 46, 2618 (1992).

[2] E.A. Sánchez, O. Grizzi, M.L. Martiarena, and V.H. Ponce, Phys. Rev. Lett. 71, 801 (1993).

[3] C.O. Reinhold, J. Burgdörfer, K. Kimura, and M. Mannami, Phys. Rev. Lett. 73, 2508 (1994).

[4] H. Lebius, R. Minniti, J.Y. Lim, and S.B. Elston, Phys. Rev. A 54, 4171 (1996).

[5] G.R. Gómez, E.A. Sánchez, O. Grizzi, M.L. Martiarena, and V.H. Ponce, Nucl. Instrum. Methods Phys. Res. B 122, 171 (1997).

[6] R. Minniti, S.B. Elston, C.O. Reinhold, J.Y. Lim, and J. Burgdörfer, Phys. Rev. A 57, 2731 (1998).

[7] G.R. Gómez, O. Grizzi, E.A. Sánchez, and V.H. Ponce, Phys. Rev. B 58, 7403 (1998).

[8] K. Kimura, S. Ooki, G. Andou, K. Nakajima, and M. Mannami, Phys. Rev. A 58, 1282 (1998); K. Kimura, G. Andou, and K. Nakajima, Phys. Rev. Lett. 81, 5438 (1998); K. Kimura, S. Ooki, G. Andou, and K. Nakajima, Phys. Rev. A 61, 012901 (1999); K. Kimura, S. Usui, and K. Nakajima, ibid. 62, 062902 (2000); K. Kimura, G. Andou, and K. Nakajima, Nucl. Instrum. Methods Phys. Res. B 164-165, 933 (2000).

[9] U. Thumm, J. Phys. B 25, 421 (1992).

[10] F.J. García de Abajo and P.M. Echenique, Phys. Rev. B 48, 13 399 (1993).

[11] C.O. Reinhold and J. Burgdörfer, Phys. Rev. A 55, 450 (1997).

[12] M.L. Martiarena, E.A. Sánchez, O. Grizzi, and V.H. Ponce, Phys. Rev. A 53, 895 (1996).

[13] M.S. Gravielle, Phys. Rev. A 58, 4622 (1998); M.S. Gravielle and J.E. Miraglia, ibid. 65, 022901 (2002).

[14] G.B. Crooks and M.E. Rudd, Phys. Rev. Lett. 25, 1599 (1970).

[15] A. Salin, J. Phys. B 2, 631 (1969).

[16] J.H. Macek, Phys. Rev. A 1, 235 (1970).

[17] G. Xiao, G. Schiwietz, P.L. Grande, A. Schmoldt, M. Grether,
R. Köhrbrük, N. Stolterfoht, A. Spieler, and U. Stettner, Nucl. Instrum. Methods Phys. Res. B 115, 215 (1996).

[18] M.S. Gravielle, Phys. Rev. A 62, 062903 (2000).

[19] D.M. Volkov, Z. Phys. 94, 250 (1935).

[20] A.B. Voitkiv and J. Ullrich, J. Phys. B 34, 4383 (2001).

[21] P.D. Fainstein, V.H. Ponce, and R.D. Rivarola, J. Phys. B 24, 3091 (1991).

[22] M.R.C. McDowell and J.P. Coleman, in Introduction to the Theory of Ion-Atom Collisions (North-Holland, Amsterdam, 1970).

[23] M.S. Gravielle and J.E. Miraglia, Comput. Phys. Commun. 69, 53 (1992).

[24] J. Burgdörfer, in Progress in Atomic and Molecular Physics, edited by C.D. Lin (World Scientific, Singapore, 1993).

[25] E. Clementi and C. Roetti, At. Data Nucl. Data Tables 14, 177 (1974).

[26] L. Hägg, C.O. Reinhold, and J. Burgdörfer, Phys. Rev. A 55, 2097 (1997).

[27] J.E. Miraglia and M.S. Gravielle (unpublished).

[28] A. Arnau, M.S. Gravielle, J.E. Miraglia, and V.H. Ponce (unpublished).

[29] J.I. Juaristi, C. Auth, H. Winter, A. Arnau, K. Eder, D. Semrad, F. Aumayr, P. Bauer, and P.M. Echenique, Phys. Rev. Lett. 84, 2124 (2000).

[30] V.G. Molière, Z. Naturforsch. 2, 133 (1947).

[31] N.R. Arista, Phys. Rev. A 49, 1885 (1994).

[32] F.J. García de Abajo and P.M. Echenique, Phys. Rev. B 46, 2663 (1992); 48, 13399 (1993).

[33] J.E. Miraglia and M.S. Gravielle, Phys. Rev. A 66, 032901 (2002).

[34] C.J. Joachain, Quantum Collision Theory (North-Holland, Amsterdam, 1979), p. 247.

[35] F. Salvat, J.M. Fernández-Varea, and M. Williamson, Jr., Comput. Phys. Commun. 90, 51 (1995). 